\title{
p75 neurotrophin receptor expression is a characteristic of the mitotically quiescent cancer stem cell population present in esophageal squamous cell carcinoma
}

\author{
TETSUJI YAMAGUCHI ${ }^{1}$, TOMOYUKI OKUMURA ${ }^{1}$, KATSUHISA HIRANO $^{1}$, TORU WATANABE ${ }^{1}$, \\ TAKUYA NAGATA $^{1}$, YUTAKA SHIMADA ${ }^{2}$ and KAZUHIRO TSUKADA ${ }^{1}$ \\ ${ }^{1}$ Department of Surgery and Science, Graduate School of Medicine and Pharmaceutical Sciences, \\ University of Toyama, Toyama City, Toyama 930-0194; ${ }^{2}$ Department of Nanobio Drug Discovery, \\ Graduate School of Pharmaceutical Sciences, Kyoto University, Sakyo-ku, Kyoto 606-8501, Japan
}

Received December 8, 2015; Accepted January 11, 2016

DOI: $10.3892 /$ ijo.2016.3432

\begin{abstract}
Mitotically quiescent cancer stem cells (CSC) are hypothesized to ex hibit a more aggressive phenotype involving greater therapeutic resistance and metastasis. The aim of our study was to develop a method for identifying quiescent CSC in esophageal squamous cell carcinoma (ESCC) based on their expression of the p75 neurotrophin receptor (p75NTR) and other proposed CSC markers, such as CD44 and CD90. Double immunostaining of surgical ESCC specimens revealed that the mean $\mathrm{Ki}-67$-labeling index of the p75NTRpositive cells was significantly lower than that of the p75NTR-negative cells. Real-time PCR analysis of sorted fractions of ESCC cell lines (KYSE cells) revealed that stem cell-related genes (Nanog, p63 and Bmi-1) and epithelialmesenchymal transition (EMT)-related genes (N-cadherin and fibronectin) were expressed at significantly higher levels in the p75NTR-positive fractions than in the CD44-positive or CD90-positive fractions. In addition, the p75NTR-positive fractions exhibited significantly higher colony formation in vitro, significantly enhanced tumor formation in mice, and significantly greater chemoresistance against cisplatin (CDDP) than the CD44-positive or CD90-positive fractions. Furthermore, in both the cultured cells and those from the mouse xenograft tumors, the p75NTR-positive/CD44negative and p75NTR-positive/CD90-negative KYSE cell fractions contained significantly higher proportions of mitotically quiescent cells. These results suggest that the mitotically quiescent CSC population in ESCC can be
\end{abstract}

Correspondence to: Dr Tomoyuki Okumura, Department of Surgery and Science, Graduate School of Medicine and Pharmaceutical Sciences, University of Toyama, 2630 Sugitani, Toyama City, Toyama 930-0194, Japan

E-mail: okumura@med.u-toyama.ac.jp

Key words: esophageal cancer, cancer stem cell, p75NTR, CD271, CD44, CD90 identified and isolated based on their p75NTR expression, providing researchers with a novel diagnostic and therapeutic target.

\section{Introduction}

Recent studies have demonstrated that some cancer stem cells (CSC) remain in a mitotically quiescent state, which enhances their chemoresistance and results in a more invasive and aggressive phenotype $(1,2)$. The p75 neurotrophin receptor (p75NTR) is expressed in the quiescent basal layer of the esophageal epithelium $(3,4)$, and in esophageal squamous cell carcinoma (ESCC). It is also expressed in populations of cells that exhibit enhanced colony-forming abilities and xenograft tumorigenicity $(5,6)$. CD44 (7) and CD90 (8) have also been reported to be markers of populations of esophageal CSC that display enhanced xenograft tumorigenicity and/or metastatic potential. However, none of these studies investigated the mitotic status of the examined CSC populations. The aim of this study was to develop a method for identifying mitotically quiescent CSC in ESCC based on their p75NTR, CD44 and CD90 expression patterns.

\section{Materials and methods}

Tissue microarray. A total of 56 tumor specimens from ESCC patients who had undergone surgery at our hospital from 1990 to 2008 were analyzed using a tissue microarray, as described previously (9). All of the patients underwent R0 resections, and none of them died in hospital. The median follow-up time was 29 months. The subjects included 50 male and 6 female patients, and their mean age was 62.8 years. The patients' TNM stages (ver. 6) were as follows: stage I, 6 patients; stage IIA, 15 patients; stage IIB, 5 patients; stage III, 24 patients; and stage IV, 6 patients. All of the M1 cases involved only distant lymph node metastases, which were surgically removed. Thirty-six patients underwent postoperative cisplatin-based chemotherapy. The institutional review board at the University of Toyama approved this study (\#20-57). 
Immunohistochemistry. The antibodies used for the immunohistochemical staining were as follows: anti-Ki-67 antibody (dilution 1:100), anti-cytokeratin Oscar in vitro diagnostic antibody (dilution 1:200; Abcam Ltd.,Cambridge, UK), anti-human CD44 monoclonal antibody (156-3C11; dilution 1:400; Cell Signaling Technology, Beverly, MA, USA), anti-human CD90 monoclonal antibody (EPR3132; dilution 1:100), and human p75NTR monoclonal antibody against p75NGER (NGER5; dilution 1:100) (both from Abcam). The immunostaining was performed using Envision Plus kits, horseradish peroxidase, or 3,3'-diaminobenzidine (DAB; Dako Cytomation, Kyoto, Japan) as recommended by the supplier. Counterstaining was performed with Mayer's hematoxylin.

The numbers of p75NTR-, CD44- or CD90-positive cells and all tumor cells were counted in three random fields of each section. Then, the proportions of each cell type in each tumor were calculated. We classified tumors as positive when $>5 \%$ of the tumor cells were stained.

Double staining of Ki-67 (brown)/p75NTR (red) or Ki-67 (brown)/CD44 (red) was performed using Bond III automated immunostainers (Leica Biosystems). The Ki-67 labeling index was defined as the number of tumor cells that exhibited positive nuclear immunostaining divided by the total number of tumor cells.

Human esophageal cancer cell lines and culture conditions. Human ESCC cell lines (KYSE-30, KYSE-140, KYSE-150, KYSE-220, KYSE-510, KYSE-520 and KYSE-790) were established by Shimada et al and cultured in Ham's F12/Roswell Park Memorial Institute (RPMI)-1640 medium (Wako, Osaka, Japan) supplemented with $2 \%$ fetal calf serum (FCS) (Gibco, Grad Island, NY, USA), according to a previously reported method (10).

Analysis of cancer stem cell surface antigens and cell sorting. The surface antigen markers of the samples were analyzed using a FACSCant II flow cytometer and were sorted using a FACSAria II cell sorter and BD FACSDiva software (both from BD Biosciences, San Jose, CA, USA). Fluorescein isothiocyanate (FITC) or allophycocyanin (APC)-conjugated monoclonal mouse anti-human CD44 (clone BD105) and p75NTR (clone ME20.4-1.H4) antibodies were purchased from Miltenyi Biotec (Bergisch Gladbach, Germany). FITC-conjugated monoclonal mouse anti-human CD90 (clone 5E-10) antibodies were obtained from BD Biosciences. FITC-conjugated antibodies were used to detect CSC surface markers. Isotype-matched APC- or FITC-conjugated antibodies (Miltenyi Biotec) were used as controls. Cultured cells were washed once with phosphate-buffered saline (PBS) (-), before being dissociated from the culture plates using $0.25 \%$ trypsin-ethylenediaminetetraacetic acid (Invitrogen, Carlsbad, CA, USA) and centrifuged. Single cells were resuspended in PBS (-) containing 2\% FBS, and then the FITC-conjugated antibody or the isotype control antibody was added, before the cells were incubated at $4^{\circ} \mathrm{C}$ for $30 \mathrm{~min}$. After being washed twice with PBS (-) containing $2 \%$ FBS, the cells were resuspended in PBS (-) containing 2\% FBS. The cells were also stained with 7-aminoactinomycin D (7-AAD; Bio-Rad Laboratories, Richmond, CA, USA) to exclude dead cells. The samples were analyzed and sorted using a flow cytometer. We
Table I. Polymerase chain reaction primer sequences.

Gene Size

product Forward and reverse primers (5'-3')

(bp)

Nanog

F: ATGCCTCACACGGAGACTGT

p63 R: AAGTGGGTTGTTTGCCTTTG

F: CAGACTTGCCAGATCATCC R: CAGCATTGTCAGTTTCTTAGC

Bmi-1

F: CCACCTGATGTGTGTGCTTTG

R: TTCAGTAGTGGTCTGGTCTTGT

Involucrin F: TGTTCCTCCTCCAGTCAATACCC R: ATTCCTCATGCTGTTCCCAGTGC

E-cadherin F: GTCTGTCATGGAAGGTGCT R: TACGACGTTAGCCTCGTTC

N-cadherin F: AGCCAACCTTAACTGAGGAGT R: GGCAAGTTGATTGGAGGGATG

Fibronectin F: AGGAAGCCGAGGTTTTAACTG R: AGGACGCTCATAAGTGTCACC

DPD

F: TCAAGCACACGACTCTTGGTG R: CATACCATTCCACAAGTCAGACC

ERCC-1 F: GCCTCCGCTACCACAACCT R: TCTTCTCTTGATGCGGCGA

GAPDH F: ACCACAGTCCATGCCATCAC
R: TCCACCACCCTGTTGCTGTA

F, forward; R, reverse.

classified cell lines as positive when $>1 \%$ of their cells were stained.

The KYSE-30 cells were sorted into p75NTR-positive/ CD44-negative, p75NTR-negative/CD44-positive, and p75NTRnegative/CD44-negative fractions. The KYSE-140 cells were sorted into p75NTR-positive/CD90-positive, p75NTR-positive/ CD90-negative, p75NTR-negative/CD90-positive and p75NTRnegative/CD90-negative fractions.

RNA extraction, cDNA synthesis andreal-time PCR. Total RNA was extracted using the TRIzol reagent (Invitrogen, Carlsbad, CA, USA). cDNA was synthesized using the PrimeScript First Strand cDNA synthesis kit (Takara Inc., Kyoto, Japan) and used for the quantitative polymerase chain reaction (qPCR) analysis. The cDNA samples were amplified in an Mx3000P real-time qPCR system (Agilent Technologies, Santa Clara, CA, USA) using SYBR Premix Ex Taq II (Takara Inc.), as described previously (11). Each mRNA expression level was normalized to that of the reference gene GAPDH. The primer sequences are shown in Table I. The expression level of each mRNA molecule was evaluated using the $\Delta \Delta \mathrm{Ct}$ method.

Cell cycle analysis. The cell cycle analysis was performed using the CycleTest Plus DNA reagent kit (Becton-Dickinson Inc., San Jose, CA, USA) as recommended by the supplier and a FACSCant II, and the resultant data were analyzed with the software FCS4 Express Cytometry (Becton-Dickinson Inc.). 

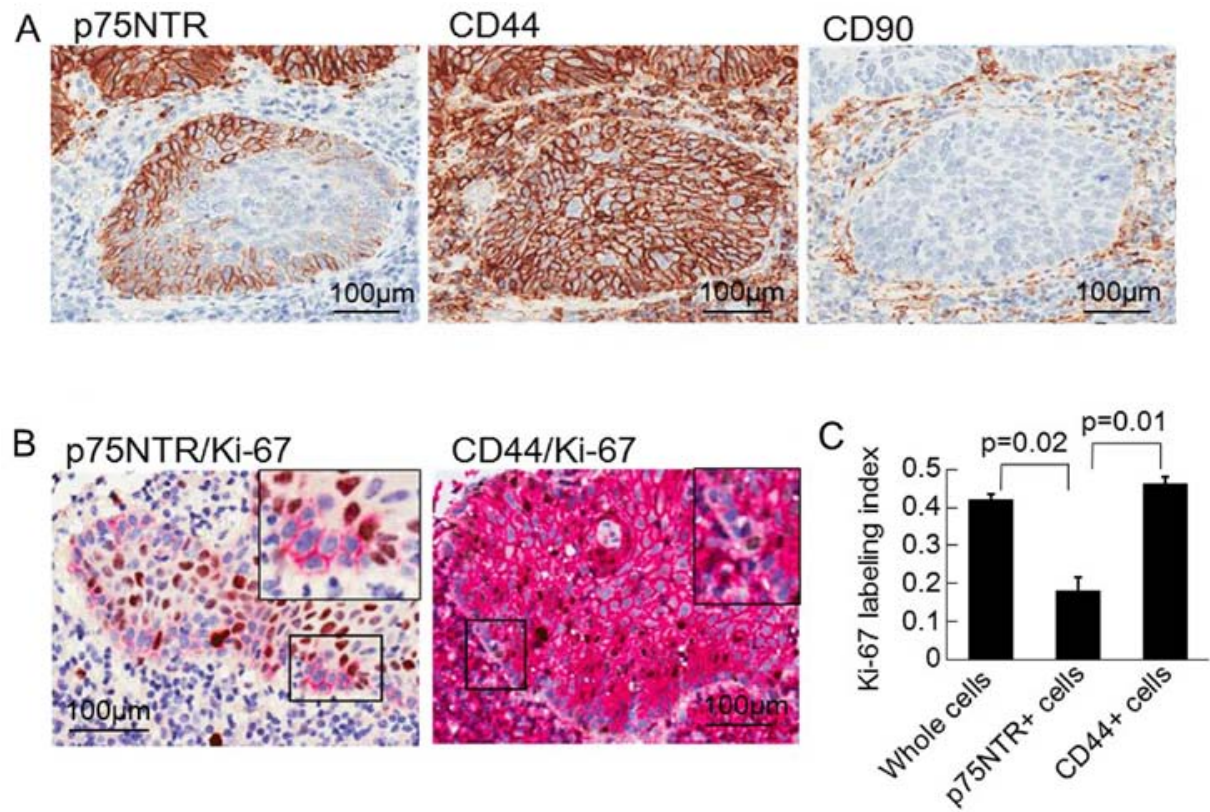

Figure 1. Expression of p75NTR, CD44 and CD90 in resected ESCC specimens. (A) The upper plates show representative findings of the immunohistochemical analysis of p75NTR, CD44 and CD90 expression in the ESCC tissue specimens. (B) The lower plates show the results of double staining of Ki-67 and p75NTR or CD44. (C) A comparison of the Ki-67 labeling indices of the p75NTR-positive cells and CD44-positive cells. The error bars represent the standard error of the mean.

Anticancer drug resistance assay. Each cell population was cultured in Dulbecco's modified Eagle's medium/Ham's F-12 containing $5 \%$ FCS at a density of 3,200 cells/well in a 96-well plate (Thermo Scientific, Yokohama, Japan) under a humidified atmosphere of $5 \% \mathrm{CO}_{2}$ at $37^{\circ} \mathrm{C}$. After being allowed to adhere overnight, the cells were treated with various concentrations of cisplatin (Wako, Osaka, Japan) or were left untreated (control). The medium in each well was changed at 2 days after the initial treatment. Cell viability was determined at 3 days after the initial treatment using the standard 3-(4,5-dimethylthiazol-2-yl)-2,5-diphenyltetrazolium bromide (MTT) assay (Trevigen, Gaithersburg, MD, USA) in accordance with the manufacturer's instructions, and the optical absorbance of the supernatant in each well at a wavelength of $595 \mathrm{~nm}$ was measured using a FilterMax F5 plate reader (Molecular Devices, Tokyo, Japan). Cell viability was defined as the relative absorbance of the control cells versus that of the treated cells. All experiments were performed in triplicate.

Colony formation assay. After being sorted with a FACSAria II, 1,000 cells from each KYSE-30 or KYSE-140 subset were plated in 60-mm tissue culture dishes (Thermo Scientific). After the cells had been cultured for 14 days, any colonies were stained with crystal violet $(0.5 \%$ crystal violet dissolved in $20 \%$ methanol). The numbers of colonies of $>3 \mathrm{~mm}$ in diameter were counted.

Tumorigenicity assay in nude mice or NOD/SCID mice. This part of the study protocol was approved by and conducted in accordance with the Committee of the Use of Live Animals in Teaching and Research at the University of Toyama. Fiveto 6-week-old mice were used for the tumorigenicity assay; athymic nude mice (BALB/CAN.Cg-Foxnl ${ }^{\mathrm{nu}} / \mathrm{CrCrlj}$ ) were used for the experiments involving KYSE-30 cells and NOD/SCID mice (NOD. CB17-Prdkc $c^{\text {scid }} / \mathbf{J}$ ) were used for those involving KYSE-140 cells. All mice were purchased from Charles River Laboratories (Yokohama, Japan). After being sorted, 1,000 to 30,000 KYSE cells were subcutaneously injected into the bilateral lumbar regions of the mice. After 4 weeks, the mice were sacrificed, and their subcutaneous tumors were fixed with $10 \%$ buffered formalin and embedded in paraffin, before being subjected to immunohistochemical staining.

In animals that were injected with cancer cells, but did not exhibit any signs of a tumor burden, the injection sites were opened up to confirm that no tumor had developed.

Statistical analysis. Statistical analyses were performed using JMP v.11 (SAS Institute Inc., Cary, NC, USA). The Chi-square test and Fisher's exact test were used for the statistical analyses, and p-values of $<0.05$ were considered to be statistically significant.

\section{Results}

Expression of p75NTR, CD44 and CD90 in the ESCC specimens. We first assessed the expression of p75NTR, CD44 and CD90 in 56 primary ESCC tumors by immunohistologically staining the tumors using a tissue microarray (Fig. 1A). p75NTR was expressed in 19 of the 56 (33.9\%) tumors, in which the first few layers nearest to the tumor's infiltrative margin exhibited positive staining. CD44 was expressed in 31 of the 56 (55.4\%) tumors and demonstrated a diffuse distribution. On the other hand, CD90 was not expressed in any of the 56 ESCC tumors despite the fact that it was detected in hepatocellular carcinoma tissue using the same procedure (data not shown). p75NTR expression was not correlated with any of the examined clinicopathological factors, such as gender, 

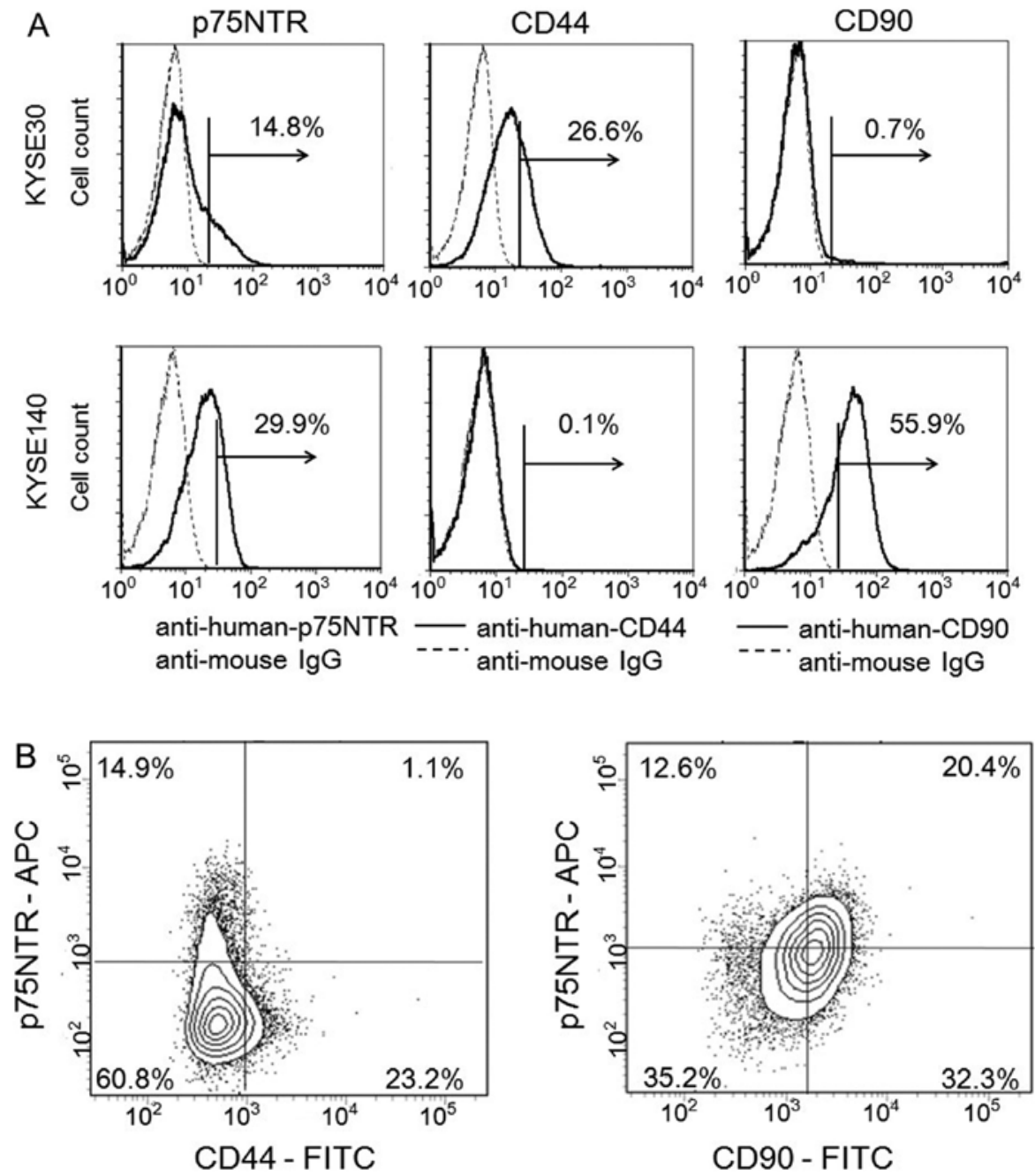

Figure 2. (A) Representative flow cytometric analysis of p75NTR, CD44 and CD90 expression in ESCC cells. Two KYSE cell lines (KYSE-30 and KYSE-140) were stained with anti-p75NTR, anti-CD44 and anti-CD90 antibodies and analyzed using flow cytometry. The levels of each marker were normalized to that of the control IgG, and the percentage of positive cells is shown in each panel. (B) KYSE-30 cells and KYSE-140 cells were co-stained with anti-p75NTR-APC and anti-CD44-FITC or anti-CD90-FITC antibodies, before being analyzed using two-color flow cytometry. The expression levels of each quadrant marker were normalized to that of the relevant isotype-matched control. The percentage of cells in each quadrant is shown.

age, tumor site, or pTNM stage (Table II), while CD44 expression was correlated with the depth of invasion and lymph node metastasis (Table II). Both p75NTR and CD44 were expressed in 12 of 56 (21.4\%) cases, although only some of the diffusely distributed CD44-positive cells were p75NTR-positive. The expression of p75NTR or CD44 alone was observed in 7 of the $56(12.5 \%)$ and 19 of the $56(33.9 \%)$ cases, respectively. Neither p75NTR nor CD44 was detected in 13 of the 56 (23.2\%) cases.

An analysis of the Ki-67 labeling index based on double immunostaining revealed that the majority of p75NTR-positive cells were in the resting phase of the cell cycle, while most of the CD44-positive cells were actively proliferating (Fig. 1B). The whole cells, the p75NTR-positive cells, and the CD44positive cells had mean Ki-67 labeling indices of $0.407,0.155$ and 0.446 , respectively (Fig. 1C).

Expression of p75NTR, CD44 and CD90 in ESCC cell lines. To identify cell subsets based on the combined expression of CSC markers in ESCC, we examined the expression of p75NTR, CD44 and CD90 in 10 ESCC cell lines (KYSE-30, KYSE-70, KYSE-140, KYSE-150, KYSE-180, KYSE-220,
KYSE-450, KYSE-510, KYSE-520 and KYSE-790) using flow cytometry (Fig. 2A and Table III). p75NTR-positive cells were detected in 6 of the $10(60.0 \%)$ cell lines, in which the proportion of positive cells ranged from 6.4 to $50.4 \%$. CD44-positive cells were detected in 9 of the $10(90.0 \%)$ cell lines, in which the proportion of positive cells ranged from 2.4 to $97.8 \%$. CD90-positive cells were detected in only 1 of the $10(10.0 \%)$ cell lines (positive proportion, 50.9\%). p75NTR and CD44, or p75NTR and CD90, were co-expressed in 5 and 1 of the 10 cell lines, respectively (Table III). Epithelial cell adhesion molecule was expressed in all of the examined ESCC cell lines, in which the proportion of positive cells ranged from 97.5 to $99.9 \%$ (data not shown). Based on the expression patterns of p75NTR, CD44 and CD90 displayed by the 10 cell lines (Table III), we selected KYSE-30 and KYSE-140 cells for the dual-color flow cytometric analysis. Among the KYSE-30 cells, which expressed p75NTR and/or CD44, p75NTR-positive/CD44-positive cells, p75NTR-positive/ CD44-negative cells, p75NTR-negative/CD44-positive cells and p75NTR-negative/CD44-negative cells accounted for 1.1, 14.9, 23.2 and $60.8 \%$ of the cells, respectively (Fig. 2B). 
Table II. Relationship between the expression of CSC markers (p75NTR, CD44 and CD90) in resected ESCC specimens and thge patient clinicopathological characteristics.

\begin{tabular}{|c|c|c|c|c|c|c|c|c|c|}
\hline & \multicolumn{3}{|c|}{ p75NTR } & \multicolumn{3}{|c|}{ CD44 } & \multicolumn{3}{|c|}{ CD90 } \\
\hline & Positive & Negative & $\mathrm{p}$-value & Positive & Negative & p-value & Positive & Negative & p-value \\
\hline \multicolumn{10}{|l|}{ Gender } \\
\hline Male & 17 & 33 & & 29 & 21 & & 0 & 50 & \\
\hline Female & 2 & 4 & 0.974 & 2 & 4 & 0.251 & 0 & 6 & - \\
\hline \multicolumn{10}{|l|}{ Age (years) } \\
\hline$\geq 65$ & 9 & 18 & & 14 & 13 & & 0 & 29 & \\
\hline$<65$ & 10 & 19 & 0.928 & 17 & 12 & 0.611 & 0 & 27 & - \\
\hline \multicolumn{10}{|l|}{ Site } \\
\hline $\mathrm{Ce}-\mathrm{Ut}$ & 3 & 5 & & 3 & 5 & & 0 & 8 & \\
\hline Mt-Ae & 16 & 32 & 0.818 & 28 & 20 & 0.276 & 0 & 48 & - \\
\hline \multicolumn{10}{|l|}{$\mathrm{pT}$} \\
\hline $\mathrm{T} 1-\mathrm{T} 2$ & 9 & 11 & & 7 & 13 & & 0 & 20 & \\
\hline T3-T4 & 10 & 26 & 0.192 & 24 & 12 & $* 0.022$ & 0 & 36 & - \\
\hline \multicolumn{10}{|l|}{$\mathrm{pN}$} \\
\hline No & 11 & 14 & & 10 & 15 & & 0 & 25 & \\
\hline N1-3 & 8 & 22 & 0.178 & 20 & 10 & $* 0.048$ & 0 & 30 & - \\
\hline \multicolumn{10}{|c|}{ Differentiation } \\
\hline Well-mod & 12 & 27 & & 22 & 17 & & 0 & 39 & \\
\hline Poor & 3 & 6 & 0.881 & 6 & 3 & 0.574 & 0 & 9 & - \\
\hline
\end{tabular}

Table III. The percentage of cells expressing CSC markers (p75NTR, CD44 and CD90) in 10 ESCC cell lines.

\begin{tabular}{lccc}
\hline & p75NTR $(\%)$ & CD44 (\%) & CD90 (\%) \\
\hline KYSE-30 & 13.8 & 30.6 & - \\
KYSE-70 & - & 97.8 & - \\
KYSE-140 & 29.9 & - & 50.9 \\
KYSE-150 & 50.4 & 7.4 & - \\
KYSE-180 & - & 2.8 & - \\
KYSE-220 & 14.9 & 2.4 & - \\
KYSE-450 & - & 3.6 & - \\
KYSE-510 & 29.9 & 97.2 & - \\
KYSE-520 & - & 80.7 & - \\
KYSE-790 & 6.4 & 3.9 & - \\
\hline
\end{tabular}

Among the KYSE-140 cells, which expressed p75NTR and/or CD90, p75NTR-positive/CD90-positive cells, p75NTRpositive/CD90-negative cells, p75NTR-negative/CD90-positive cells and p75NTR-negative/CD90-negative cells accounted for 20.4, 12.6, 32.3 and 35.2\% cells, respectively (Fig. 2B).

Expression of CSC-related genes in fractionated cell subsets. We compared the expression patterns of stem cell-, keratinocyte differentiation- and epithelial-mesenchymal transition (EMT)-related genes in each fraction using real-time PCR. Among the KYSE-30 cells, Nanog, p63 and
Bmi-1 were expressed at significantly higher levels in the p75NTR-positive/CD44-negative fraction than in the p75NTRnegative/CD44-positive or p75NTR-negative/CD44-negative fraction (Fig. 3A). In addition, the expression of involucrin was significantly lower in the p75NTR-positive/CD44-negative fraction than in the p75NTR-negative/CD44-positive or p75NTR-negative/CD44-negative fraction (Fig. 3A).

The p75NTR-positive/CD44-negative and p75NTRnegative/CD44-positive fractions demonstrated significantly lower E-cadherin expression than the p75NTRnegative/CD44-negative fraction (Fig. 3C). In addition, the p75NTR-positive/CD44-negative fraction displayed significantly higher $\mathrm{N}$-cadherin and fibronectin expression than the p75NTR-negative/CD44-positive and p75NTR-negative/CD44-negative fractions (Fig. 3C).

Among the KYSE-140 cells, Nanog, p63 and Bmi-1 were expressed at significantly higher levels in the p75NTR-positive/CD90-positive and p75NTR-positive/CD90-negative fractions than in the p75NTR-negative/CD90-positive or p75NTR-negative/CD90-negative fraction (Fig. 3B). The p75NTR-negative/CD90-negative fraction exhibited significantly higher involucrin expression than the other fractions (Fig. 3B). Significantly greater E-cadherin expression was observed in the p75NTR-negative/CD90-negative fraction than in the p75NTR-positive/CD90-positive, p75NTRpositive/CD90-negative or p75NTR-negative/CD90-positive fraction (Fig. 3D). The p75NTR-positive/CD90-positive fraction exhibited significantly higher $\mathrm{N}$-cadherin and fibronectin expression than the other fractions (Fig. 3D). 
A
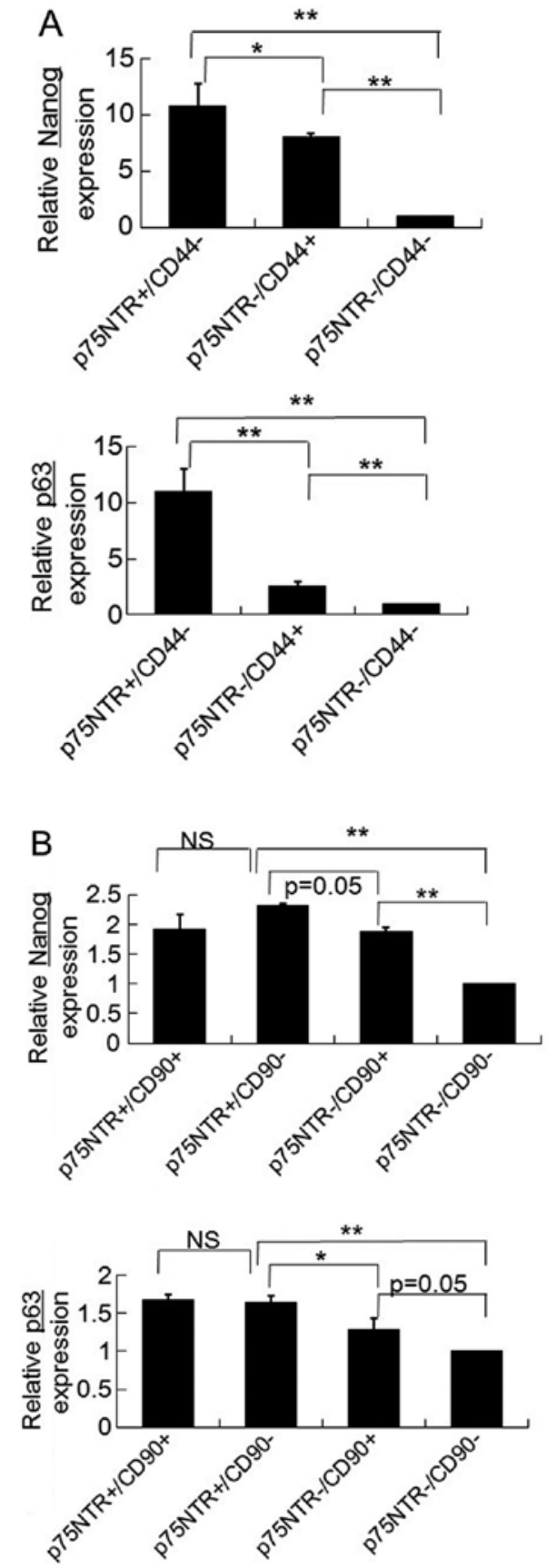
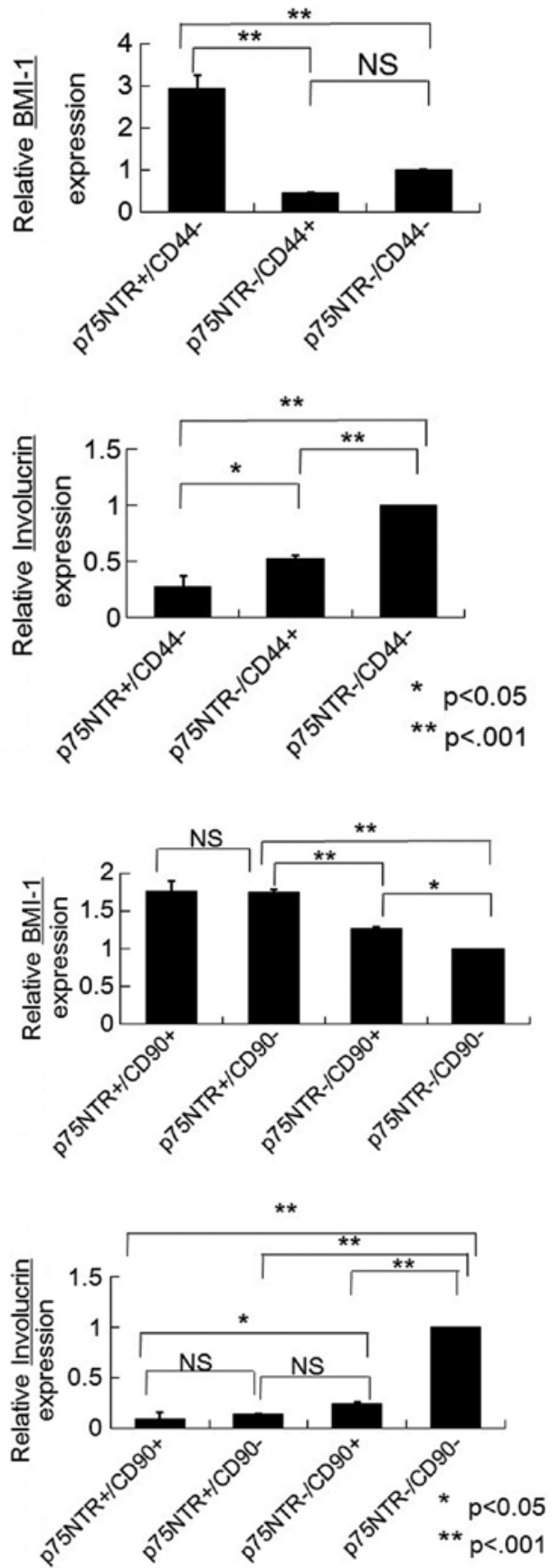

Figure 3. Expression of CSC-related genes in each cell subset. (A and B) The CSC-related mRNA and keratinocyte differentiation-related mRNA expression levels in sorted p75NTR-positive/CD44-positive cells isolated from KYSE-30 cells (A) or sorted p75NTR-positive/CD90-positive cells isolated from KYSE-140 cells (B) were measured by real-time PCR. The expression levels of these cell markers are shown relative to their expression levels in the p75NTR-negative/CD44-negative cells or p75NTR-negative/CD90-negative cells. The error bars represent the standard error of the mean.

Cell cycle analysis. Flow cytometric cell cycle analysis revealed that the p75NTR-negative/CD44-positive KYSE-30 cells were a mitotically active subpopulation and exhibited the lowest proportion of cells in the G0/G1 phase. On the other hand, the p75NTR-positive/CD44-negative and p75NTR-negative/CD44-negative fractions were relatively quiescent cell fractions, in which $>67 \%$ of the cells were in the G0/G1 phase (Fig. 4A). Among the KYSE-140 cells, the p75NTRpositive/CD90-positive fraction contained the most actively proliferating cells, while the p75NTR-positive/CD90-negative fraction was composed of quiescent cells, $42.6 \%$ of which were in the G0/G1 phase (Fig. 4B).
Comparison of drug-resistant ability. We compared the drug resistance of each cell population using the MTT assay. Among the KYSE-30 cells, the p75NTR-negative/CD44-positive cells exhibited significantly lower viability than the p75NTRpositive/CD44-negative cells after being treated with CDDP (Fig. 4C). Among the KYSE-140 cells, the p75NTRnegative/CD90-negative cells displayed significantly lower viability than the p75NTR-positive or CD90-positive cells after being treated with CDDP (Fig. 4C).

In vitro colony formation ability. The ability of the KYSE-30 cells to form colonies exhibited the following 
C
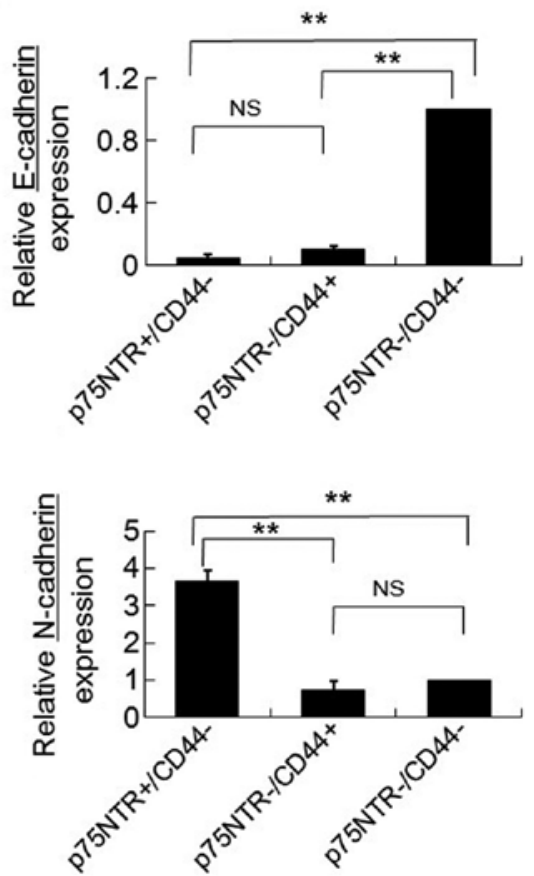

$\mathrm{D}$
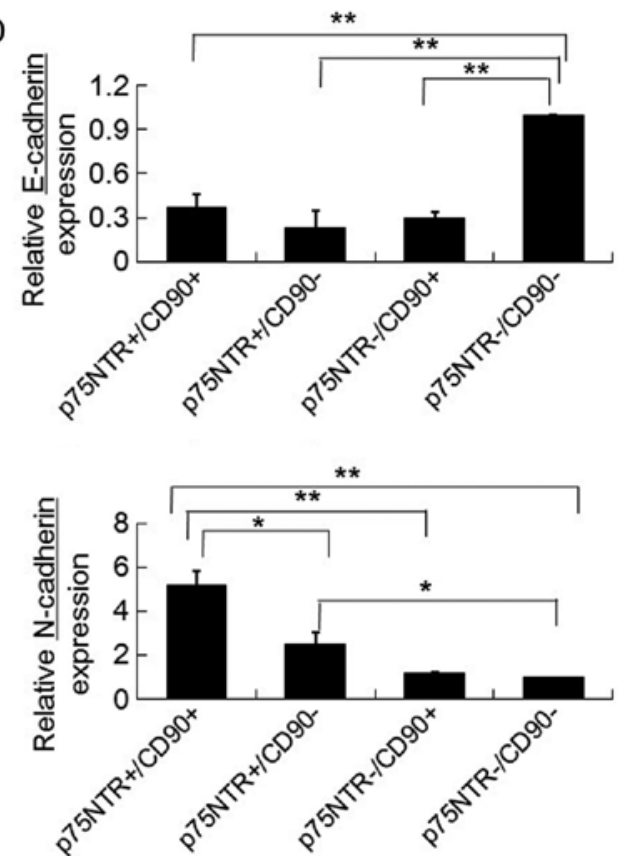

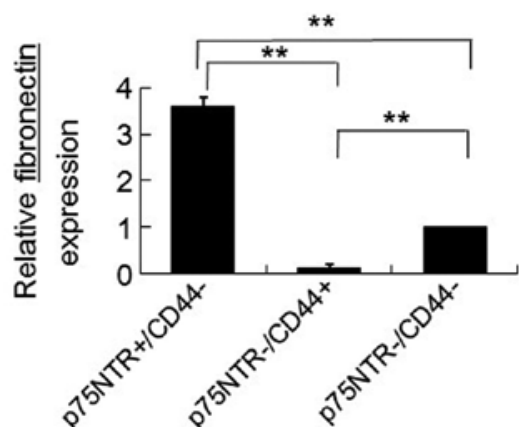

* $p<0.05$

${ }^{* *} p<.001$

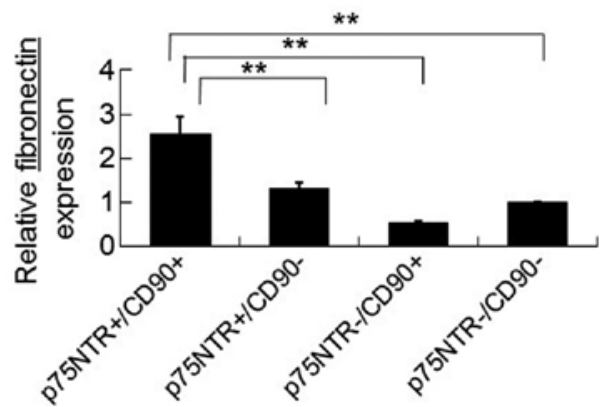

* $\mathrm{p}<0.05$

$* * p<001$

Figure 3. Continued. (C and D) EMT-related mRNA expression levels in sorted p75NTR-positive/CD44-positive cells isolated from KYSE-30 cells (C) or sorted p75NTR-positive/CD90-positive cells isolated from KYSE-140 cells (D) were measured by real-time PCR. The expression levels of these cell markers are shown relative to their expression levels in the p75NTR-negative/CD44-negative cells or p75NTR-negative/CD90-negative cells. The error bars represent the standard error of the mean.

order: p75NTR-positive/CD44-negative > p75NTR-negative/ CD44-positive > p75NTR-negative/CD44-negative (Fig. 5A). As for the KYSE-140 cells, their ability to form colonies displayed the following order: p75NTR-positive/ CD90-negative > p75NTR-positive/CD90-positive > p75NTR-negative/CD90-positive > p75NTR-negative/CD90negative (Fig. 5A).

In vivo tumor formation. Among the KYSE-30 cells, the subcutaneous injection of as few as $3 \times 10^{3}$ p75NTR-posi-
tive/CD44-negative cells or p75NTR-negative/CD44-positive cells into nude mice resulted in the development of tumors at $4 / 4(100 \%)$ and $2 / 4(50 \%)$ of the injection sites, respectively, at 4 weeks after the injection procedure. In contrast, the injection of $1 \times 10^{4}$ p75NTR-negative/CD44-negative cells did not result in tumor formation (Table IV).

The tumors that developed after the injection of $1 \times 10^{4}$ p75NTR-positive/CD44-negative cells weighed significantly more than those derived from the p75NTR-negative/ CD44-positive cells (Fig. 5B). Histological examinations 


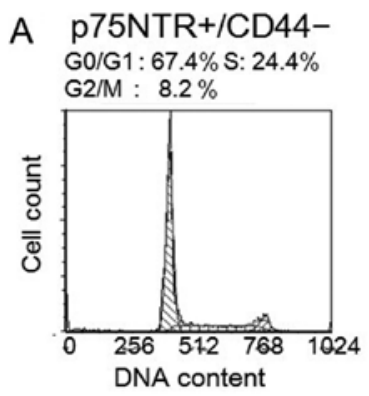

p75NTR-/CD44-

G0/G1: $69.2 \%$ S: $22.9 \%$

$\mathrm{G} 2 / \mathrm{M}: 8.0 \%$

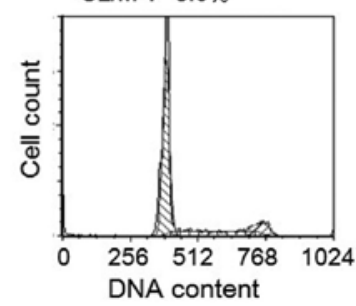

B p75NTR+/CD90+ $\mathrm{G} 2 / \mathrm{M}: 42.0 \%$

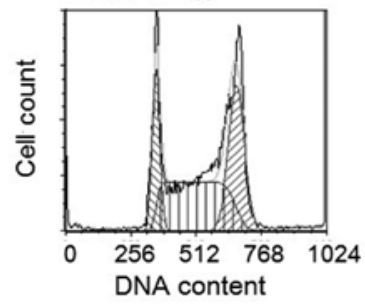

DNA content

p75NTR-/CD90+

Go/G1: $31.3 \%$ S : $52.9 \%$ $\mathrm{G} 2 / \mathrm{M}: 15.8 \%$

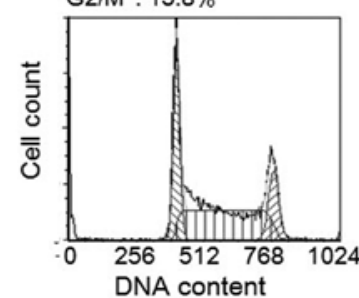

p75NTR-/CD44+

G0/G1: $45.3 \%$ S: $40.3 \%$

$\mathrm{G} 2 / \mathrm{M}: 14.5 \%$

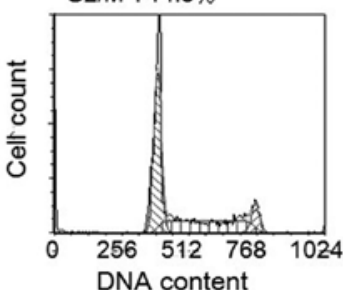

DNA content

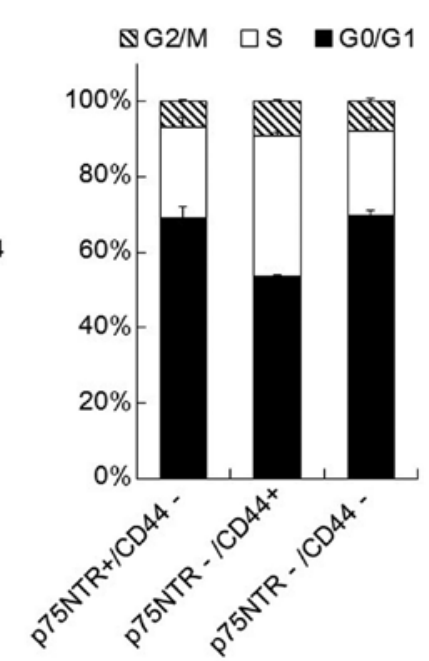

p75NTR+/CD90 -

G0/G1: $42.6 \%$ S : $44.7 \%$

$\mathrm{G} 2 / \mathrm{M}: 12.7 \%$

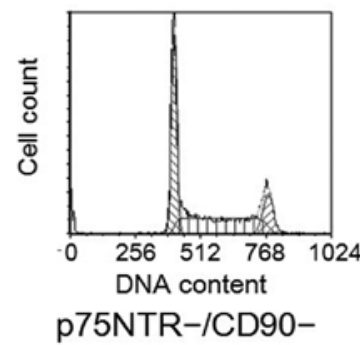

$\mathbb{N G} / \mathrm{M} \square \mathrm{S} \square \mathrm{GO} / \mathrm{G} 1$

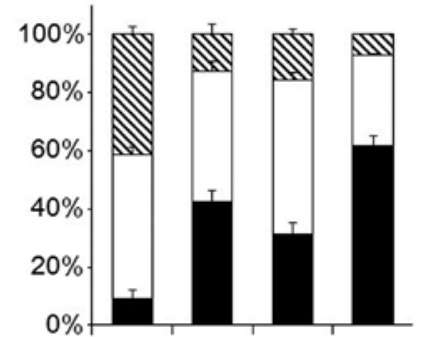
$\mathrm{G} 2 / \mathrm{M}: 15.8 \%$
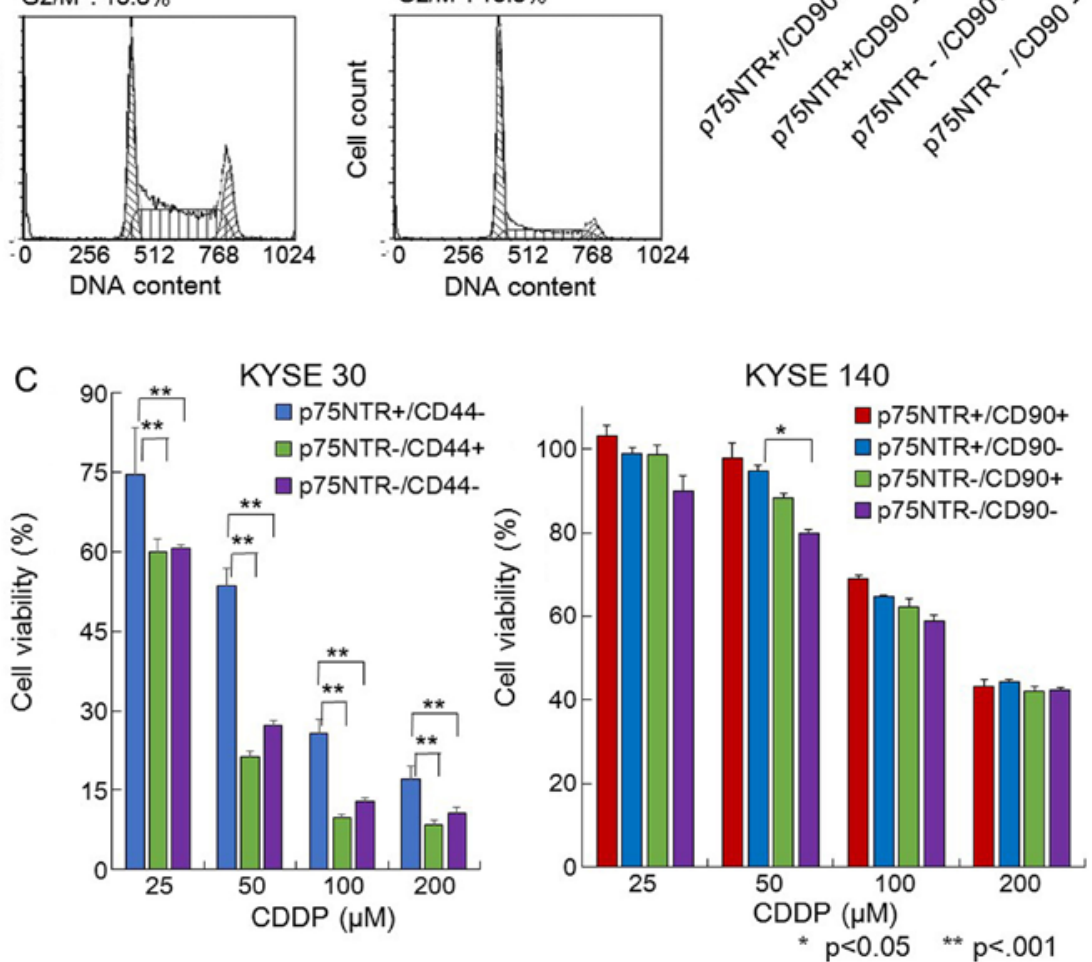

Figure 4. The cell cycle distribution and chemoresistance of KYSE-30 and KYSE-140 cell subsets. (A and B) Approximately $2 \times 10^{4}$ live cells were analyzed using the CycleTest Plus DNA reagent kit and a FACSCant II. The results were then analyzed using FCS4 express cytometry software. The proportion of cells in each phase of the cell cycle is shown in each panel. The error bars represent the standard error of the mean. (C) Fractionated KYSE-30 and KYSE-140 cells in 96 -well plates $(3,200$ cells/well) were treated with cisplatin $(25,50,100$ or $200 \mu \mathrm{M})$ (or left untreated) for 3 days, and then their viability was determined using the MTT assay. 
A
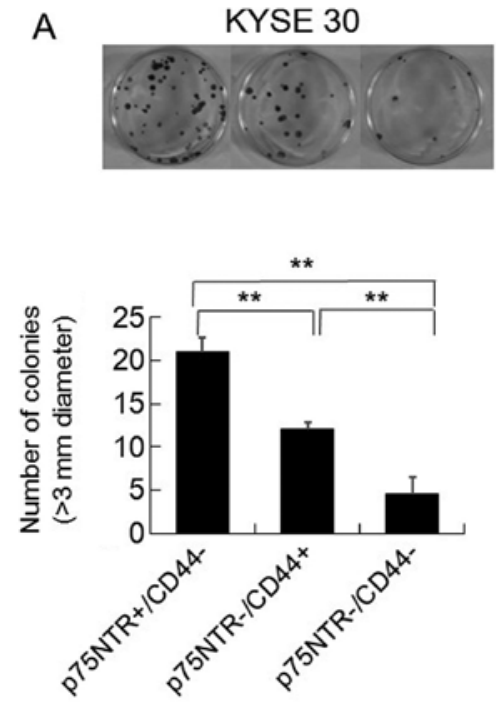

B
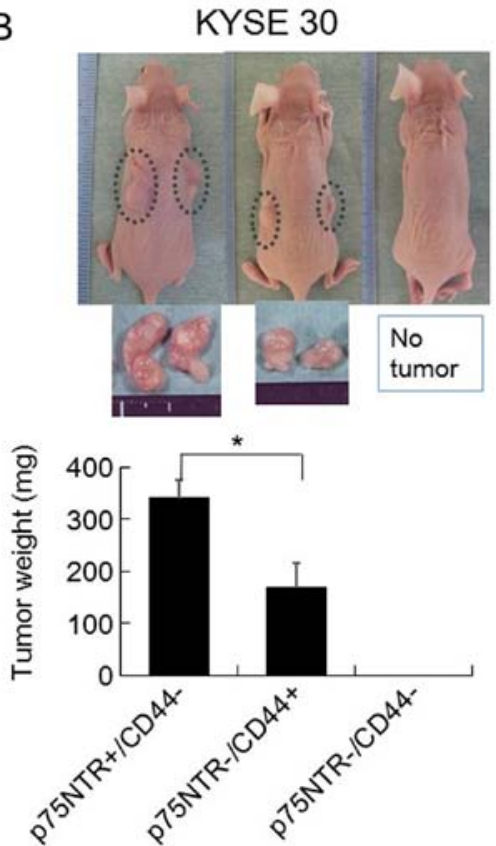

KYSE 140
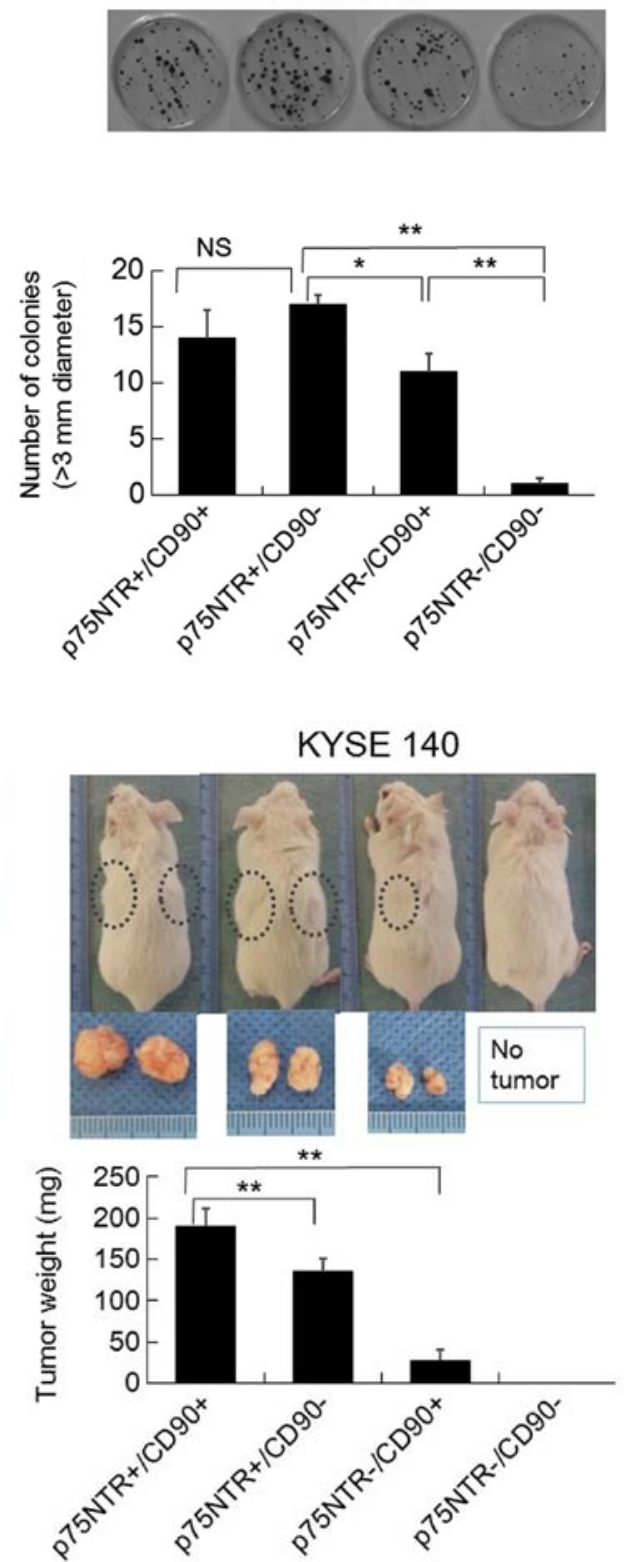

Figure 5. Colony formation assay and tumorigenicity analysis. (A) The colony formation ability of subpopulations of KYSE-30 and KYSE-140 cells that were isolated based on their p75NTR, CD44 and CD90 expression patterns. Crystal violet staining of the colonies that had formed at 2 weeks after the plating procedure (upper panels). The mean number of colonies of $>3 \mathrm{~mm}$ diameter was compared. The error bars represent the standard error of the mean (lower panels). (B) Representative nude mice with subcutaneous tumors derived from 1x10 ${ }^{4}$ of p75NTR-positive/CD44-negative, p75NTR-negative/CD44-positive, or p75NTR-negative/CD44-negative KYSE-30 cells and subcutaneous tumors derived from these subpopulations of KYSE-30 cells (left upper panels). Representative NOD/SCID mice with subcutaneous tumors derived from 1x104 p75NTR-positive/CD90-negative, p75NTR-positive/CD90-negative, p75NTRnegative/CD90-positive, or p75NTR-negative/CD90-negative KYSE-140 cells, and subcutaneous tumors derived from this subpopulation of KYSE-140 cells (right upper panels). The lower panels shows the mean tumor weight at each site. The error bars represent the standard error of the mean.

of the xenograft tumors demonstrated that their phenotypes resembled those of primary ESCC tumors (Fig. 5C). p75NTR was expressed in a small number of the cancer cells in the first few layers nearest to the tumor infiltrating margins. CD44 was diffusely expressed throughout the tumors, except in the cells residing at their margins. A detailed examination of $4 \mu \mathrm{m}$-thick serial sections detected p75NTR-positive/CD44-negative cells at the tumor infiltrating margins. Analyses of the Ki-67 labeling index based on double immunostaining revealed that $86.9 \%$ of the p75NTR-positive cells were in the resting phase of the cell cycle, while $74.8 \%$ of the CD44-positive cells were actively proliferating.
Among the KYSE-140 cells, the subcutaneous injection of as few as $3 \times 10^{3}$ p75NTR-positive/CD90-positive cells or p75NTR-positive/CD90-negative cells into NOD/SCID mice resulted in tumors forming at $2 / 4(50 \%)$ and $1 / 4(25 \%)$ of the injection sites, respectively, at 4 weeks after the injection procedure (Table IV).

On the other hand, the injection of $1 \times 10^{4}$ p75NTR-negative/CD90-positive cells resulted in the establishment of tumors at $2 / 4(50 \%)$ of the injection sites. In contrast, even the injection of $3 \times 10^{4}$ p75NTR-negative/CD90-negative cells did not result in tumor formation (Table IV). The tumors that developed after the injection of $1 \times 10^{4}$ p75NTR-positive/CD90-positive 

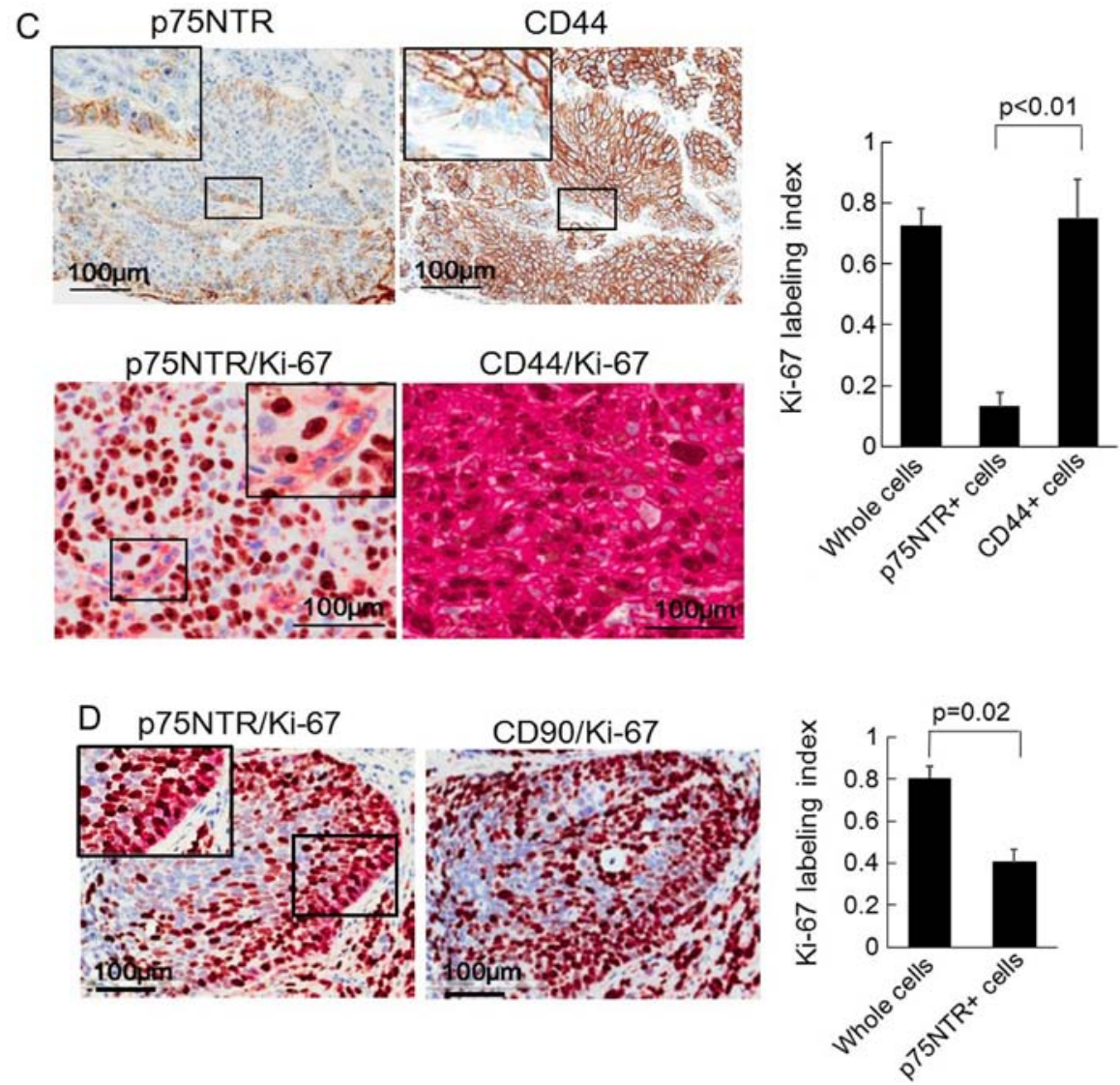

Figure 5. Continued. (C) Representative immunohistochemical findings obtained during the staining of p75NTR and CD44 in a tumor derived from p75NTRpositive/CD44-negative KYSE-30 cells (upper panels), and the lower panels show the results obtained during the double staining of Ki-67 and p75NTR or CD44. Ki-67 labeling index data are also shown. (D) Representative immunohistochemical findings obtained during the double staining of Ki67-NTR and p75NTR or CD90 in a tumor derived from p75NTR-positive/CD90-positive KYSE-140 cells. Ki-67 labeling index data are also shown (right panel).

Table IV. In vivo tumor development experiment in which p75NTR-positive/CD44-positive KYSE-30 cells were injected into nude mice and p75NTR-positive/CD90-positive KYSE-140 cells were injected into NOD/SCID mice.

\begin{tabular}{|c|c|c|c|c|}
\hline & \multirow[b]{2}{*}{ No. of cells injected } & \multicolumn{3}{|c|}{ Tumor incidence } \\
\hline & & 30,000 & 10,000 & 3,000 \\
\hline \multirow[t]{3}{*}{ KYSE-30 } & $\mathrm{p}^{2} 5 \mathrm{NTR}^{+} / \mathrm{CD}_{4} 4^{-}$ & $2 / 2$ & $4 / 4$ & $4 / 4$ \\
\hline & p75NTR $/ \mathrm{CD} 44^{+}$ & $2 / 2$ & $4 / 4$ & $2 / 4$ \\
\hline & p75NTR $/$ CD44- & $2 / 2$ & $0 / 4$ & $0 / 4$ \\
\hline \multirow[t]{4}{*}{ KYSE-140 } & p75NTR ${ }^{+} / \mathrm{CD}_{90}{ }^{+}$ & $2 / 2$ & $2 / 2$ & $2 / 4$ \\
\hline & $\mathrm{p}^{2} 5 \mathrm{NTR}^{+} / \mathrm{CD} 90^{-}$ & $2 / 2$ & $4 / 4$ & $1 / 4$ \\
\hline & p75NTR $/ \mathrm{CD} 90^{+}$ & $2 / 2$ & $2 / 4$ & $0 / 4$ \\
\hline & p75NTR $/$ CD90- & $0 / 2$ & $0 / 4$ & $0 / 4$ \\
\hline
\end{tabular}

cells weighed significantly more than those derived from the p75NTR-positive/CD90-negative $(\mathrm{p}<0.001)$ or p75NTRnegative/CD90-positive cells $(\mathrm{p}<0.001)$ (Fig. 5B). In addition, the tumors derived from the p75NTR-positive/CD90-negative cells were larger than those derived from the p75NTR-negative/CD90-positive cells ( $\mathrm{p}=0.002$ ) (Fig. 5B). A histological examination of the xenograft tumors showed that p75NTR was expressed in the cancer cells at their infiltrating margins. An analysis of the Ki-67 labeling index based on double immunostaining revealed that $59.6 \%$ of the p75NTR-positive cells were in the resting phase of the cell cycle, while $80.4 \%$ of the tumor cells were actively proliferating. No CD90 expression was detected in any of the cancer cells in the xenograft tumors $(\mathrm{p}=0.017)($ Fig. 5D) .

\section{Discussion}

Our immunohistochemical investigation of surgical ESCC specimens for previously reported CSC markers, such as p75NTR, CD44 and CD90, revealed that the expression of p75NTR alone, the expression of CD44 alone, and the expression of both p75NTR and CD44 were detected in 21.4, 25.0 and $30.4 \%$ of the tumors, respectively. In addition, none of the reported CSC markers were detected in $23.2 \%$ of the tumors, demonstrating the heterogeneity of the cell surface marker expression of ESCC tumors, as has been shown for various other types of tumors (12).

p75NTR was expressed at the tumor infiltrating margins, while CD44 was diffusely expressed throughout the tumors, which is compatible with the findings of previous studies $(5,7)$. Our double immunostaining-based analysis of the Ki-67 labeling index revealed that most of the p75NTR-positive cells were in a mitotically quiescent state, while the majority of the CD44-positive cells were actively proliferating. These findings indicated that quiescent CSC can be found within 
ESCC and that it may be possible to identify different CSC populations based on the combination of cell surface markers that they express.

No CD90 expression was detected in any of the tumor cells in the ESCC specimens. However, we observed positive CD90 staining in the stromal cells surrounding the cancer cells in all of the ESCC specimens, which is compatible with the findings of a previous report, in which CD90 expression was detected in the stromal cells of head and neck squamous cell carcinoma (13). We also observed positive CD90 staining in the tumor cells of hepatocellular carcinoma specimens (positive controls) (7). Although a previous study detected CD90 expression in ESCC specimens by qPCR and isolated CD90positive cells from fresh cancer tissues by flow cytometry (8), no previous studies have detected CD90 expression in tumor cells from ESCC specimens.

p75NTR, CD44 and CD90 expression were detected at various cell frequencies in 6,9 and 1 of the 10 examined KYSE cell lines, respectively, further indicating the heterogeneity of the cell surface marker expression seen in ESCC.

Contrary to the finding we obtained during the immunohistochemical examination of the ESCC specimens, CD90 was expressed in $50.9 \%$ of the KYSE-140 cells. It has been reported that CD90 expression can be induced by various biological agents, such as thymopoietin, prostaglandins, interleukin-1, tumor necrosis factor- $\alpha$, vascular endothelial growth factor, and nerve growth factor (NGF) $(14,15)$. Combining the above-mentioned finding with the fact that an NGF autocrine loop was detected in KYSE cells (16), it is suggested that CD90 expression can be induced in certain cell culture conditions.

Among the KYSE-30 cells, the p75NTR-positive CD44negative fraction exhibited strong stem cell-related gene expression, weak keratinocyte differentiation marker expression, anticancer drug resistance, in vitro colony formation and in vivo tumorigenicity; i.e., they displayed a CSC phenotype. Furthermore, the majority of the p75NTR-positive CD44negative KYSE-30 cells were in a mitotically quiescent state, indicating that a quiescent CSC population is maintained even in actively proliferating cells, whereas, the CD44-positive cells were actively proliferating and exhibited a less marked CSC phenotype.

Among the KYSE-140 cells, the p75NTR-positive cells displayed a CSC phenotype; i.e., strong stem cell-related gene expression, weak keratinocyte differentiation marker expression, anticancer drug resistance, in vitro colony formation, and in vivo tumorigenicity, regardless of whether they expressed CD90.

Furthermore, an analysis of the KYSE-140 cells based on their p75NTR/CD90 expression patterns identified two distinct subpopulations in terms of their cell cycle status. The p75NTR-positive/CD90-negative subset was mitotically quiescent cells while the p75NTR-positive/CD90-positive subset was actively proliferating cells, further indicating the existence of a quiescent CSC population in ESCC.

Recent studies have demonstrated that dormant CSC are even present in solid tumors, such as melanoma (17) and ovarian (18), breast (2) and pancreatic tumors (19). In addition, it was reported that these cells enhance chemoresistance, invasiveness and metastasis, as well as the risk of a late relapse after curative surgery $(1,2,12,20)$.
Several studies of leukemia have examined the molecular mechanisms that regulate stem cell quiescence. These studies suggested that inducing leukemia cells to enter the cell cycle may enhance their chemosensitivity and that it is important to identify molecules that will enable us to directly target quiescent CSC $(21,22)$.

Recently it was reported that the NGF/proNGF/p75NTR axis plays a critical role in regulating the self-renewal of quiescent CSC in breast cancer (23). Combining our results with the findings of a previous report in which NGF overexpression and its autocrine loop was shown to enhance cell proliferation in KYSE cells (16), it is possible that p75NTR signaling also plays a role in the regulation of quiescent CSC in ESCC.

We used negative immunohistochemical staining of the proliferation marker Ki-67 and flow cytometric measurements of DNA content to detect cells in the G0/G1 phase of the cell cycle, as it was previously reported that hematopoietic stem cells exist in the quiescent state (according to dual staining using the DNA-binding dye Hoechst 33342 and anti-Ki-67 antibody) (24). Moreover, label incorporation-based assays, such as 5-bromo-2-deoxyuridine (BrdU)-based studies, have also been used to isolate putative quiescent CSC based on their dynamic cycling kinetics (25). Further studies of selfrenewal, asymmetric cell division and the duration of the $\mathrm{G} 0 /$ G1 phase in the p75NTR-positive cell subset may facilitate the elucidation of the dynamic kinetics of the CSC present in ESCC.

In the present study, we found that EMT markers were strongly expressed in the p75NTR-positive/CD44-negative and p75NTR-positive/CD90-positive fractions of the KYSE-30 and KYSE-140 cells, respectively. Previous studies have demonstrated that EMT processes play a role not only in the acquisition of a migratory mesenchymal phenotype, which enhances invasiveness and metastasis, but also in the acquisition of stem cell and tumorigenic characteristics in CSC $(20,26)$. Combining these results with the findings of a recent report in which it was demonstrated that NGF promoted EMT in the breast cancer stem cell compartment (23), it is possible that p75NTR signaling is involved in the regulation of EMT and stem cell properties. The identification of the molecular mechanisms that regulate cell stem cell properties, cell cycle status and EMT processes in the p75NTR-positive cell subset of KYSE cells may provide a basis for the development of novel treatment strategies for ESCC.

In conclusion, by examining surgical tumor specimens and cultured cell lines we found that ESCC exhibit heterogeneous expression patterns of cell surface markers such as p75NTR, CD44 and CD90. In addition, an analysis of the Ki-67 labeling indices in the tumor specimens revealed that most of the p75NTR-positive cells were in a mitotically quiescent state, while the majority of the p75NTR-negative cells were actively proliferating. Among KYSE cells, p75NTR-positive cells represent a CSC population that exhibits strong stem cell-related gene expression, weak keratinocyte differentiation marker expression, chemoresistance, in vitro colony formation, and in vivo tumorigenicity, regardless of whether they express CD44 or CD90.

Furthermore, p75NTR-positive/CD44-negative and p75NTR-positive/CD90-negative fractions KYSE cells were found to be mitotically quiescent CSC both in vitro and in vivo. 
These results demonstrate that it is possible to detect and isolate quiescent CSC from ESCC, providing researchers with a target that will aid the development of novel therapeutic strategies, as well as diagnostic tools for patient selection.

\section{Acknowledgements}

The authors wish to thank Mr. Masahiko Kawahara for performing the cell culture experiments and providing technical assistance. This study was supported by a Grant-in-Aid for Scientific Research (C) from MEXT KAKENHI (grant no. 15K10088). This study was also supported by C-CAM.

\section{References}

1. Li L and Bhatia R: Stem cell quiescence. Clin Cancer Res 17: 4936-4941, 2011.

2. Pece S, Tosoni D, Confalonieri S, Mazzarol G, Vecchi M, Ronzoni S, Bernard L, Viale G, Pelicci PG and Di Fiore PP: Biological and molecular heterogeneity of breast cancers correlates with their cancer stem cell content. Cell 140: 62-73, 2010.

3. Okumura T, Shimada Y, Imamura $M$ and Yasumoto $S$ : Neurotrophin receptor p75(NTR) characterizes human esophageal keratinocyte stem cells in vitro. Oncogene 22: 4017-4026, 2003.

4. Okumura T, Shimada Y, Sakurai T, Hori R, Nagata T, Sakai Y and Tsukada K: Abnormal cell proliferation in the p75NTR-positive basal cell compartment of the esophageal epithelium during squamous carcinogenesis. Dis Esophagus 28: 634-643, 2015.

5. Okumura T, Tsunoda S, Mori Y, Ito T, Kikuchi K, Wang TC, Yasumoto $\mathrm{S}$ and Shimada Y: The biological role of the low-affinity p75 neurotrophin receptor in esophageal squamous cell carcinoma. Clin Cancer Res 12: 5096-5103, 2006.

6. Huang SD, Yuan Y, Liu XH, Gong DJ, Bai CG, Wang F, Luo JH and Xu ZY: Self-renewal and chemotherapy resistance of p75NTR positive cells in esophageal squamous cell carcinomas. BMC Cancer 9: 9, 2009.

7. Zhao JS, Li WJ, Ge D, Zhang PJ, Li JJ, Lu CL, Ji XD, Guan DX, Gao $\mathrm{H}, \mathrm{Xu} \mathrm{LY}$, et al: Tumor initiating cells in esophageal squamous cell carcinomas express high levels of CD44. PLoS One 6: e21419, 2011

8. Tang KH, Dai YD, Tong M, Chan YP, Kwan PS, Fu L, Qin YR, Tsao SW, Lung HL, Lung ML, et al: A CD90(+) tumor-initiating cell population with an aggressive signature and metastatic capacity in esophageal cancer. Cancer Res 73: 2322-2332, 2013.

9. Shimada Y, Okumura T, Sekine S, Moriyama M, Sawada S, Matsui K, Yoshioka I, Hojo S, Yoshida T, Nagata T, et al: Expression analysis of iPS cell - inductive genes in esophageal squamous cell carcinoma by tissue microarray. Anticancer Res 32: 5507-5514, 2012.

10. Shimada Y, Imamura $M$, Wagata $T$, Yamaguchi $N$ and Tobe $T$ : Characterization of 21 newly established esophageal cancer cell lines. Cancer 69: 277-284, 1992.

11. Sekine S, Shimada Y, Nagata T, Moriyama M, Omura T, Yoshioka I, Hori R, Matsui K, Sawada S, Okumura T, et al: Establishment and characterization of a new human gallbladder carcinoma cell line. Anticancer Res 32: 3211-3218, 2012.
12. Dick JE: Stem cell concepts renew cancer research. Blood 112: 4793-4807, 2008.

13. Liotta F, Querci V, Mannelli G, Santarlasci V, Maggi L, Capone M, Rossi MC, Mazzoni A, Cosmi L, Romagnani S, et al: Mesenchymal stem cells are enriched in head neck squamous cell carcinoma, correlates with tumour size and inhibit T-cell proliferation. Br J Cancer 112: 745-754, 2015.

14. Chung MT, Liu C, Hyun JS, Lo DD, Montoro DT, Hasegawa M, Li S, Sorkin M, Rennert R, Keeney M, et al: CD90 (Thy-1)-positive selection enhances osteogenic capacity of human adipose-derived stromal cells. Tissue Eng Part A 19: 989-997, 2013.

15. Haeryfar SMM and Hoskin DW: Thy-1: More than a mouse pan-T cell marker. J Immunol 173: 3581-3588, 2004.

16. Tsunoda S, Okumura T, Ito T, Mori Y, Soma T, Watanabe G, Kaganoi J, Itami A, Sakai Y and Shimada Y: Significance of nerve growth factor overexpression and its autocrine loop in oesophageal squamous cell carcinoma. Br J Cancer 95: 322-330, 2006.

17. Roesch A, Fukunaga-Kalabis M, Schmidt EC, Zabierowski SE, Brafford PA, Vultur A, Basu D, Gimotty P, Vogt T and Herlyn M: A temporarily distinct subpopulation of slow-cycling melanoma cells is required for continuous tumor growth. Cell 141: 583-594, 2010.

18. Gao MQ, Choi YP, Kang S, Youn JH and Cho NH: CD24+ cells from hierarchically organized ovarian cancer are enriched in cancer stem cells. Oncogene 29: 2672-2680, 2010.

19. Dembinski JL and Krauss S: Characterization and functional analysis of a slow cycling stem cell-like subpopulation in pancreas adenocarcinoma. Clin Exp Metastasis 26: 611-623, 2009.

20. Mani SA, Guo W, Liao M-J, Eaton EN, Ayyanan A, Zhou AY, Brooks M, Reinhard F, Zhang CC, Shipitsin M, et al: The epithelial-mesenchymal transition generates cells with properties of stem cells. Cell 133: 704-715, 2008.

21. Roeder I, Horn M, Glauche I, Hochhaus A, Mueller MC and Loeffler M: Dynamic modeling of imatinib-treated chronic myeloid leukemia: Functional insights and clinical implications. Nat Med 12: 1181-1184, 2006.

22. Zhang B, Strauss AC, Chu S, Li M, Ho Y, Shiang KD, Snyder DS, Huettner CS, Shultz L, Holyoake T, et al: Effective targeting of quiescent chronic myelogenous leukemia stem cells by histone deacetylase inhibitors in combination with imatinib mesylate. Cancer Cell 17: 427-442, 2010.

23. Tomellini E, Touil Y,Lagadec C, Julien S, Ostyn P,Ziental-Gelus N, Meignan S, Lengrand J, Adriaenssens E, Polakowska R, et al: Nerve grow th factor and proNGF simultaneously promote symmetric self-renewal, quiescence, and epithelial to mesenchymal transition to enlarge the breast cancer stem cell compartment. Stem Cells 33: 342-353, 2015.

24. Wilson A, Laurenti E, Oser G, van der Wath RC, Blanco-Bose W, Jaworski M, Offner S, Dunant CF, Eshkind L, Bockamp E, et al: Hematopoietic stem cells reversibly switch from dormancy to self-renewal during homeostasis and repair. Cell 135: 1118-1129, 2008.

25. Buczacki S, Davies RJ and Winton DJ: Stem cells, quiescence and rectal carcinoma: An unexplored relationship and potential therapeutic target. Br J Cancer 105: 1253-1259, 2011.

26. Chaffer CL, Marjanovic ND, Lee T, Bell G, Kleer CG, Reinhardt F, D'Alessio AC, Young RA and Weinberg RA: Poised chromatin at the ZEB1 promoter enables breast cancer cell plasticity and enhances tumorigenicity. Cell 154: 61-74, 2013. 